Running Head: LATENT PROFILE AND LATENT CLASS ANALYSIS

\title{
A PRIMER TO LATENT PROFILE AND LATENT CLASS ANALYSIS
}

\author{
Johannes Bauer
}

University of Erfurt

University of Erfurt

Nordhäuser Str. 63

99089 Erfurt

Germany,

Phone: +49 361 737-2025

Fax: +49 $361737-2019$

E-mail: johannes.bauer@uni-erfurt.de

ORCID: 0000-0001-6801-2540

This is a preprint of the following chapter: Johannes Bauer, "A Primer to Latent Profile and Latent Class Analysis", published in "Methods for Researching Professional Learning and Development: Challenges, Applications and Empirical Illustrations", edited by Michael Goller, Eva Kyndt, Susanna Paloniemi, and Crina Damşa, 2022, Springer, reproduced with permission of Springer. The final authenticated version is available online at: https://link.springer.com/book/10.1007/978-3-031-08518-5 


\begin{abstract}
This chapter gives an applied introduction to latent profile and latent class analysis (LPA/LCA). LPA/LCA are model-based methods for clustering individuals in unobserved groups. Their primary goals are probing whether and, if so, how many latent classes can be identified in the data, and to estimate the proportional size and response profiles of these classes in the population. Moreover, latent class membership can serve as predictor or outcome for external variables. Substantively, LPA/LCA adopt a person-centered approach that is useful for analyzing individual differences in prerequisites, processes, or outcomes of learning. The chapter provides a conceptual overview of LPA/LCA, a nuts-and-bolts discussion of the steps and decisions involved in their application, and illustrative examples using freely available data and the R statistical environment.
\end{abstract}

Keywords: Latent variable models, mixture models, latent class analysis, latent profile analysis. 


\section{Introduction}

Suppose you are a lecturer in a study program who is teaching a compulsory lecture on a difficult topic. The exam consists of a standardized test that is well-established in your department and that provides scores ranging between 0 and 50. To get an impression of your students' achievement in the recent years you analyze the available data $(N=1000)$. You are surprised to find that the data do not follow a normal distribution, as you would have expected, but a bi-modal one, as illustrated in Figure 1a. Since you are wondering whether something is wrong with the test you discuss this finding with a colleague. She mentions that the distribution looks like the data came from two separate groups with different achievement levels. Indeed, reportedly some students try passing the exam by rote rehearsal whereas others prepare more thoroughly applying deeper learning strategies. Given the test tasks' nature, it is reasonable that rote rehearsal leads to diminished performance. Unfortunately, you do not have data on students' learning strategies. All you have is the achievement data. Nevertheless, you wonder whether it would be possible to identify the unobserved groups given the data.

Let us put the latter question aside for a moment and suppose now we have insight of the true population characteristics, as illustrated in Figure 1b. Indeed, there are two subpopulations, the low-achieving students (supposedly superficial learners) marked red and the high achieving ones (supposedly deep learners) marked blue. Because these subpopulations are unobserved in our empirical data they are hidden from us and called latent classes. Within each latent class, achievement differences follow a normal distribution with class-specific (conditional) means and standard deviations. The bi-modal cross-class distribution is a marginal combination of these two within-class distributions and, therefore, called a mixture distribution. 

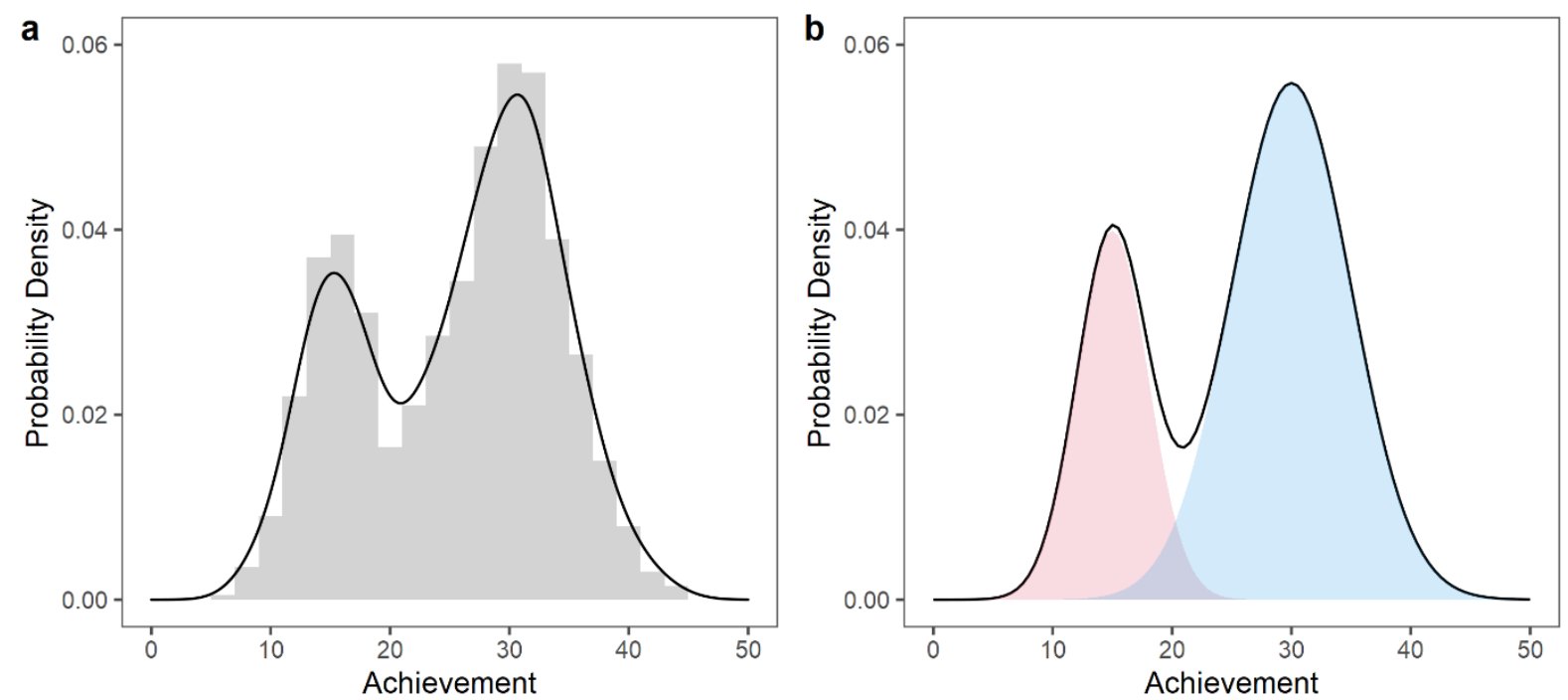

Fig. 1. Illustrative univariate finite normal mixture distribution: (a) empirically observed distribution $(N=1000)$; (b) true population distributions: two within-class distributions [red: $\mathcal{N}(\mu=15 ; \sigma=3)$; blue: $\mathcal{N}(\mu=30 ; \sigma=5)]$ and their joint mixture distribution (black line).

So, how can we recover the latent classes shown in Figure $1 \mathrm{~b}$ based on the observed achievement data? This is where mixture models come in. Mixture models are a family of statistical procedures for uncovering hidden groups. As Oberski (2016) put it, "Mixture modeling is the art of unscrambling eggs" (p. 275). In this chapter, I will introduce latent profile analysis (LPA) and latent class analysis (LCA) which are mixture models for cross-sectional data. The main difference between them is that LPA applies to continuous response variables, whereas LCA applies to categorical ones. As we will see, a LPA for the example data in Figure la gives us information on (i) the number of latent classes, (ii) model fit and the accuracy of the classification, (iii) the proportional size of each class, (iv) the within-class means and variances, and (v) probability values for each person's latent class membership. Of course, since we have not observed class membership, the classification is inevitably uncertain. A major advantage of LPA/LCA over traditional cluster analysis is that this classification uncertainty is an explicit part of the statistical model (Rost, 2003; Vermunt \& Magidson, 2002). 
The following section will give readers a conceptual background of LPA/LCA. Next, I provide didactic examples how to implement LPA and LCA. The chapter will close with some cautionary considerations, a note on software, and recommendations for further reading.

\section{Conceptual overview}

\subsection{Family relations}

As mentioned, LPA/LCA belong to the broad family of mixture models (Hancock \& Samuelsen, 2008; Mclachlan \& Peel, 2000; Masyn, 2013; Sterba, 2013). The common purpose of all mixture models is to provide a probabilistic classification of persons to latent classes based on a statistical model, where each class is one of the $K$ categories of a discrete latent variable $c$. Hence, unlike in traditional factor analysis or IRT modeling, the latent variable is categorical instead of continuous. All mixture models combine a within-class and a betweenclass model (Sterba, 2013). The within-class model defines the population parameters for persons in a given class $k$ who constitute a relatively homogeneous sub-group regarding these parameters. The between-class model defines each persons' probability of being sampled from the sub-population underlying class $k$. Thus, mixture models cluster persons who are similar in terms of the within-class parameters and yield per person a classification probability for each class. Persons then can be assigned to a latent class based on their highest membership probability (modal class assignment). This probability-based (soft) clustering is a major conceptual advantage of mixture models over the deterministic (hard) assignment applied in traditional cluster analysis because it explicitly accounts for the mentioned classification uncertainty (Mair, 2018; Rost, 2003; Vermunt \& Magidson, 2002).

Differences among various kinds of mixture models concern the parameters that are in focus of the classification (i.e., conditional means/item probabilities vs. regression parameters) and whether the data are cross-sectional vs. longitudinal (Tab. 1). Moreover, the observed items' level of scale can be considered, e.g., to distinguish LPA and LCA. The latter distinction is somewhat artificial, however, since both LPA and LCA are just special cases of the general 
mixture model. It is even possible to combine indicators of mixed levels of scale in the same analysis (Vermunt \& Magidson, 2002; see Mair, 2018, for an illustrative application).

Table 1. Overview of selected mixture models.

\begin{tabular}{|c|c|c|}
\hline & \multicolumn{2}{|c|}{ Classification by } \\
\hline \multirow[b]{2}{*}{ Design } & Means (continuous data) / & \\
\hline & item probabilities (categorical data) & Regression parameters \\
\hline \multirow[t]{2}{*}{ Cross-sectional } & Latent profile analysis & Regression mixture models \\
\hline & Latent class analysis & \\
\hline \multirow[t]{2}{*}{ Longitudinal } & Repeated measures latent class analysis & Growth mixture models \\
\hline & Latent transition analysis & \\
\hline
\end{tabular}

As indicated in Table 1, latent classes in LPA/LCA are characterized by qualitatively different response profiles on the analyzed items (Rost, 2003; Vermunt \& Magidson, 2002). If the items are continuous, the profiles are patterns of class-specific means; if they are categorical, the profiles are defined by the items' class specific endorsement probabilities (i.e., for dichotomous items the probability of scoring 1 vs. 0 ). This basic idea extends to the longitudinal case. For example, repeated measures LCA models classes of mean/probability-trajectories across multiple measurement occasions; latent transition analysis models latent classes for each time point (latent statuses) as well as transitions between them (Collins \& Lanza, 2010). Though not elaborated in this chapter, mixture models for regression coefficients are noteworthy because they allow uncovering differential treatment effects in experimental or intervention research (Jung \& Wickrama, 2008; Oberski, 2016).

As a final note, mixture models can be used both in an exploratory, hypothesis-generating mode of data analysis and in a confirmatory, hypothesis-testing one (Finch \& Bronk, 2011). The former occurs more frequently in practice and applies when a researcher has only tentative 
or no prior assumptions about the latent classes. It should be emphasized, however, that even confirmatory LPA/LCA cannot test hypotheses about a specific number of classes. As elaborated below, this number is not an estimable model parameter. Testable hypotheses concern only the class sizes and their response patterns on the items.

\subsection{The LPA and LCA models}

LPA. In LPA, we typically assume a normal distribution within each latent class, though other distributions are possible, such as Poisson for count data (Mair, 2018; Vermunt \& Magidson, 2002). Note that the normality assumption applies only to these subpopulation distributions, not to their joint distribution, and it is untestable. Each latent class $(1, \ldots, K)$ has a density $f_{k}\left(x_{i} \mid \mu_{k}, \sigma_{k}^{2}\right)$, where $x_{i}$ is an observed value for person $i$ on a continuous indicator $m$, and $\mu_{k}$ and $\sigma_{k}^{2}$ are its mean and variance in class $k$. The joint mixture model is given by

$$
f\left(x_{i}\right)=\sum_{k=1}^{K} \pi_{k} f_{k}\left(x_{i} \mid \mu_{k}, \sigma_{k}^{2}\right)
$$

where $\pi_{k}$ is the relative class size, or equivalently, the class probability (also called mixture weight). That is, each person's density in the mixture distribution is a sum of the class specific normal densities weighted by the class probabilities. For example, consider persons with a testscore of 21 in Figure 1b. Their mixture density (i.e., value on the black curve) is the sum of the two class-specific densities (i.e., values on the red and blue curves, respectively) which are weighted by the respective class probabilities. In the multivariate case, where $\boldsymbol{x}_{\boldsymbol{i}}$ is a vector of responses on a set of $M$ observed items, the model remains essentially the same but each class has its specific mean vector $\boldsymbol{\mu}_{\boldsymbol{k}}$ and variance-covariance matrix $\boldsymbol{\Sigma}_{\boldsymbol{k}}$.

Of course, before the analysis, we neither know the class probabilities nor the class-specific means and (co)variances. Unless further restrictions are imposed, these are the model parameters that have to be estimated. Because the number of free parameters grows quickly with the number of latent classes and indicators, it makes sense to specify further restrictions to increase model parsimony and estimation stability (Vermunt \& Magidson, 2002). Frequently, 
one imposes a local independence restriction meaning that the indicators are constrained to be uncorrelated within each latent class. ${ }^{1}$ Additionally, a cross-class equality restriction on the indicator variances can be imposed delivering homogeneity of the variance-covariance matrices across classes (Vermunt \& Magidson, 2002). Together these two restrictions ensure that the classes differ only in their mean profiles, and not in the form of the variance-covariance matrix, which facilitates interpretation (Peugh \& Fan, 2013). Several other models with different sets of restrictions are available (Masyn, 2013; Pastor et al., 2007; Scrucca et al., 2016). Finally, an important point visible in equation 1 is that the number of classes is not an estimated model parameter. Rather, the number of classes has to be decided upon by the analyst on grounds of statistical and substantive criteria (see section 2.3). Once a target model has been selected, its estimated parameters can be interpreted.

LCA. The LCA model works similarly though it applies to categorical items. LCA starts with the basic idea of local independence, that is, it seeks to establish latent classes such as that responses on a set of categorical items are independent within classes (Rost, 2003). Suppose we have a vector of responses $\boldsymbol{u}_{\boldsymbol{i}}$ to a set of $M$ categorical items. Under the local independence assumption, the LCA model provides the probability for a given response pattern on these items by

$$
P\left(\boldsymbol{u}_{\boldsymbol{i}}\right)=\sum_{k=1}^{K} \pi_{k} \prod_{m=1}^{M} P\left(u_{m i} \mid c_{i}=k\right) .
$$

That is, the probability of a specific combination of item responses depends on the class probabilities and the product of the item response probabilities given class membership. These are the model parameters to be estimated. For items with $J$ categories the item probabilities are the $J-1$ thresholds between these categories. Again, further restrictions may be imposed on the

\footnotetext{
${ }^{1}$ That is, residual correlations among the indicators are zero given class membership. Local independence is a default assumption in many latent variable models, such as factor analysis or IRT models, but can be relaxed.
} 
model. Note that the local independence assumption can be evaluated and also be partially relaxed in LCA (Asparouhov \& Muthén, 2015; Masyn, 2013).

\subsection{Steps in LPA/LCA}

Conducting an LPA/LCA is an iterative, stepwise process similar to exploratory factor analysis. Four steps can be distinguished, the last of which is optional: (i) specifying the model, (ii) determining the number of latent classes, (iii) interpreting the target solution substantively, and (iv) including predictors and/or outcomes of latent class membership. In the following, I briefly elaborate on each of these steps.

Step 1: Model specification. In specifying the model researchers need to make a theorybased selection of observed indicators, decide how these indicators enter the model, and what, if any, restrictions to impose on the model parameters. Concerning the selection of indicators, one should keep in mind that LPA/LCA are measurement models (Wang \& Wang, 2020). So, there should be a rationale that the indicators measure a joint theory-based construct (e.g. different profiles of self-concept). In practice, LPA/LCA is also frequently applied to classify persons over a range of conceptually related, but distinct constructs (e.g., J. Bauer \& Mulder, 2013; Gillet et al., 2020). In that case, the resulting profiles are mere summaries of persons with similar answer patterns and should not be interpreted as realizations of a latent trait (see section 4.1 on the danger of reification). In either case, it makes only sense to apply LPA/LCA if the set of indicators is inter-correlated. So, screening item associations prior to the analysis is warranted.

A further consideration in LPA is whether to include indicators on the item level or in aggregated form, and whether to apply prior transformations. Aggregation is frequently done by computing composite mean scores, factor scores, or (preferably) plausible values of the (sub-)scales to be analyzed (e.g., Bauer \& Prenzel, 2021; Hong et al., 2020; Kunst et al., 2016; 
Mair, 2018). ${ }^{2}$ This approach considerably reduces the number of parameters to be estimated and better approximates continuous indicators. An arguably optimal approach in this regard is second-order LPA in which the latent class variable is a second-order variable with continuous first-order factors serving as indicators (for an application see Bauer et al., 2018). With this specification, the indicators used for the latent classification are latent factors that are truly continuous and corrected for measurement error. Note that this approach requires scaling the first-order factors to have a meaningful mean structure (Little et al., 2006).

Concerning parameter restrictions, researchers may wish to start with the local independence model since this is the traditional basis of LCA, and potentially consider crossclass equality constraints on the indicator variances. Any other restrictions should be made on substantive grounds (see Rost, 2006, for interesting applications). Attention should be payed to the default restrictions implemented by specific software packages. For example, Mplus (Muthén \& Muthén, 1998-2017) by default imposes local independence and homogeneity across classes. An alternative strategy implemented routinely in the mclust package (Scrucca et al., 2016) is estimating a variety of models with different restrictions and choosing the best fitting one (including a decision on the number of latent classes; see Step 2). This data driven strategy alleviates researchers from a priori considerations about restrictions. However, interpreting what the restrictions applied by the selected model mean substantively may not always be straightforward. For further details, see section 3.2.

Step 2: Class enumeration. Deciding on the number of classes may be the most difficult step in the whole analysis. The goal is to yield a solution that balances model parsimony and fit, and that delivers substantively interpretable classes. For this purpose, one estimates ${ }^{3}$ a series

\footnotetext{
${ }^{2}$ Using composite scores of categorical items as indicators will turn a LCA into a LPA.

${ }^{3}$ Discussing details of estimation is beyond the scope of this chapter; for a tractable introduction, see Masyn (2013).
} 
of models from 1 to $K$ latent classes and compares them on indices of relative model fit and classification diagnostics. Then, the candidate model(s) are considered substantively.

The most commonly used fit indices are the Bayesian Information Criterion (BIC), its sample-size-adjusted variant (SABIC), and the Akaike Information Criterion (AIC). With these and any other information criteria, lower values indicate better model fit. Ideally, a minimum value in the series of candidate models points at the best-fitting solution. In practice, fit indices may continue to improve as more latent classes are extracted. In such circumstances, an elbowplot - similar to the scree-plot in exploratory factor analysis - can be useful to identify at which step the improvement in model fit plateaus. Next to information criteria, models with adjacent numbers of latent classes can be compared by a likelihood ratio test (LRT) which is available in several versions. Some simulation studies indicate the bootstrapped LRT (BLRT) to be quite effective in class enumeration (Nylund et al., 2007). A statistically significant result of the LRT supports the $k$-class model over $k$ - 1 classes. In theory, one choses the model after which the LRT is statistically non-significant for the first time. Again, in practice the LRT may continue to stay significant for the whole series of probed models, especially with large sample sizes.

Classification diagnostics can additionally inform the class enumeration process. For each latent class, average posterior class probabilities $\left(A v e P P_{k}\right)$ provide a measure of classification accuracy. As the name suggests, they are based on averaging for class $k$ the model-estimated (i.e. posterior) latent class membership probabilities for individuals with their highest membership probability in this class. Values $\geq .70$ have been proposed as desirable (Masyn, 2013). The information about classification accuracy is condensed in the entropy measure that ranges between 0 and 1 and can be interpreted similar to a reliability coefficient for the classification (values $\geq .80$ are desirable, $\geq .60$ a suggested minimum; Asparouhov \& Muthén, 2014). Classification accuracy may be helpful to decide between solutions that have similar fit (Rost, 2006), but note that entropy is not a good sole or primary model selection criterion (Masyn, 2013). 
As a final word of caution, though there is extensive research on fit indices in mixture models (e.g., Nylund et al., 2007; Peugh \& Fan, 2013; Tofighi \& Enders, 2008), results have not yet converged and there is no single best index. Applied researchers frequently combine an array of indices for class enumeration but - probably more often than not - their results may prove to be inconsistent. Therefore, there is general agreement in the methodological literature that model selection needs to be guided at least as much by the substantive interpretability of the class solution as by fit indices. If multiple solutions with similar fit occur, one should inspect all of them and make a decision on substantive grounds.

Step 3. Substantive interpretation of the target model(s). The interpretation process in LPA/LCA is similar to traditional cluster analysis or exploratory factor analysis. For this purpose, one inspects the class-specific mean/probability profiles across the indicators and differences in these profiles across classes. Depending on model specification, class specific (co)variances are of importance, too. Based on this interpretation, a definition and label need to be attached to each class. Finally, the class sizes are of interest to distinguish common or normative profiles from more exceptional ones. In general, no solution should be retained that is not well interpretable, regardless of model fit. Some authors consider a solution useless in which not all classes are interpretable (Wang \& Wang, 2020). However, Mair (2018) notes that the occurrence of a single uninterpretable junk-class is not problematic since it may simply contain the persons that did not fit in any of the other classes.

Though interpretation is primarily a theoretical issue, some methodological considerations apply (Collins \& Lanza, 2010; Masyn, 2013; Rost, 2006): First, the class profiles should be distinct enough. That is, there should be low variability within classes and a high one between them (Collins \& Lanza, 2010). In this context, it may be helpful to compare solutions with adjacent numbers of classes to see from what classes in a prior solution members of a new class are drawn (Rost, 2006). Sometimes a class in the prior solution is simply split up in two classes and the researcher needs to consider the value added by this differentiation. Moreover, finding 
the structure of profiles to vary greatly across different solutions may be indicative that LPA/LCA is not an appropriate model for the data (Rost, 2006).

Second, it should be checked, whether the indicators can be arranged in a way so that the class profiles are ordered (i.e., they do not cross each other in a profile plot). Such a solution particularly, if the profiles are more or less parallel - would indicate that the classes differ by degree, rather than by type (Rost, 2006; Wang \& Wang, 2020). In such a case, the classification is still meaningful, but a model focusing on quantitative differences in the latent variable (i.e., a factor or IRT model) would provide a more parsimonious account of the data.

Finally, solutions with several small classes (say $\leq 5 \%$ ) may be indicative that too many classes have been extracted (Nylund et al., 2007). Though such small classes can be of substantive interest, it should be checked critically whether they have a distinct and interpretable profile.

Step 4: Include predictors and distal outcomes of most likely latent class membership. Having established a latent class solution, a logical next step is to analyze predictors or dependent variables of latent class membership (Vermunt \& Magidson, 2002). This is called LPA/LCA with covariates and distal outcomes. Such analyses are useful (i) to enrich the description of the latent classes based on background information (e.g., social background variables or experimental condition), (ii) to analyze the construct-validity of the latent class solutions, or (iii) to test hypotheses for answering substantive research questions. The latter two cases lead to a blend of exploratory and confirmatory analyses (Masyn, 2013) because the hypotheses can only be stated after the classification. However, even if the classification is intrinsically exploratory, the later hypotheses about the classes' relation to covariates and distal outcomes can still be tested in a confirmatory fashion.

Methods for analyzing covariates and distal outcomes in mixture models are still an active area of research. Though it may be tempting to simply extract individuals' modal class assignment from the results and use this as a manifest variable for further analyses (e.g. as factor 
in an ANOVA), it is well known that this procedure leads to biased results and is inappropriate (Bakk \& Kuha, 2020). The reason is that this hard classification disregards the classification uncertainty. A variety of alternative approaches has been developed. Recent overviews recommend using the two-step method or one of the bias-corrected three-step methods $(\mathrm{BCH}$ and ML) for handling covariates; (manual) $\mathrm{BCH}$ is advisable for continuous and categorical distal outcomes (Asparouhov \& Muthén, 2021; Bakk \& Kuha, 2020; Nylund-Gibson et al., 2019). Unfortunately, these approaches are currently only available in commercial software (Latent Gold, Mplus). Illustrating the application of LPA/LCA with covariates and distal outcomes is beyond the scope of this chapter, but readers will find didactic examples in the literature cited above as well as in Wang and Wang (2020).

\subsection{A person-centered conceptual perspective}

So far, this chapter has introduced LPA/LCA from a statistical perspective. What, however, is the substantive added value of mixture models - and LPA/LCA in particular - for studying professional learning and development? Mixture models have unique advantages in studying individual differences in learning, its conditions, and outcomes (Bauer et al., 2018; Collins \& Lanza, 2010; Hancock \& Samuelsen, 2008; Masyn, 2013). Conceptually, mixture analyses adopt a person-centered approach that is helpful when a researcher aims to analyze categorical individual differences in terms of a classification of persons according to typical patterns of their characteristics or behavior (Bergman \& Magnusson, 1997). This person-centered perspective is complementary to the traditional variable-centered one that aims at identifying relationships between variables. For example, taking a variable-centered perspective, researchers might be interested in the degree to which some predictors are relevant for explaining employees' engagement in work-related learning activities. From a person-centered perspective they may ask what typical patterns of engagement in learning activities occur, how prevalent these patterns are, and what background variables characterize people demonstrating a specific pattern. Hence the two perspectives are complementary and should not be 
dichotomized falsely (Masyn, 2013). Synergies can arise from taking both perspectives on the same research topic (Marsh et al., 2009). For example, Bauer and Mulder (2013) studied predictors of and individual differences in nurses' engagement in learning activities after errors at work. In a variable-centered analysis, they found that nurses' perceptions of errors and their social context predicted this engagement. A subsequent LPA complemented these findings by identifying four typical patterns of reactions to errors and learning engagement, with a substantial proportion of nurses $(>40 \%)$ showing dysfunctional behavior.

More generally speaking, LPA/LCA can be used whenever there are theoretical reasons to believe that a latent construct is categorical. As Collins and Lanza (2010) argue, many variables of substantive interest can be understood both in terms of continua and categories. They employ the example of alcohol consumption which may be defined continuously as the volume of alcohol consumed in a given period of time. At the same time, it makes substantive sense to distinguish categories of alcohol consumption, such as non-drinking, occasional drinking, social drinking, binge drinking, or being alcohol addict. Referring to our introductory example, test achievement is arguably a continuous variable, but it can be meaningful to distinguish learners showing mastery vs. non-mastery. So, readers will find that LPA/LCA (and other mixture models) are applicable to a broad spectrum of variables relevant to professional and work-related learning.

\section{Implementation of LPA and LCA}

This section will provide three didactic examples of LPA and LCA. We will use simulated data and the packages mclust (Scrucca et al., 2016), tidyLPA (Rosenberg et al., 2018), and poLCA (Linzer \& Lewis, 2011) in the R statistical environment (R Core Team, 2021) so that readers can reproduce all analyses. Though the data are simulated, Examples 2 and 3 are based on published studies that used LPA (Gillet et al., 2020) and LCA (Richter et al., 2013). Below, I restrict the presentation to the most important parts of the in- and output. Complete code and data files are available in the supplemental online material (SOM) at https://osf.io/x3ahn. 


\subsection{Example 1: Univariate LPA of student achievement}

Turning back to the initial example from Figure 1, we will use LPA to recover the two hidden groups in the mixture distribution of student achievement. Since there is only one variable, our only choice in model specification is whether we allow the class-specific variances to vary, or not. Since our empirical data suggest different spread in the presumed clusters (Fig. 1a) it seems reasonable to freely estimate the variances within each class.

For class enumeration, we estimate models with $k=1$ to 4 latent classes using the code pasted below. The argument modelName = "V" specifies varying variances across classes. If we omit this argument, mclust will estimate models with and without equality constraints and users can compare their fit to the data. The next commands call for an elbow plot of BIC (see SOM) and the BLRT.



Unfortunately, mclust delivers only a few fit indices. If we desire a broader variety of indices, we can compute them manually based on the information stored in the output object m1. Otherwise, we may get enhanced output by using the functions estimate_profiles() and get_fit() from tidyLPA which provides a set of wrapper functions for mclust. Both options are demonstrated in the SOM. Table 2 presents a selection of fit indices, all of which indicate that the two-class model fits the data best. This is backed up by the BLRT showing that the two-class model fits the data significantly better than the oneclass model, whereas moving to three classes does not provide a further statistically significant improvement in fit. 
Table 2. Selected fit indices for the univariate LPA of student achievement.

\begin{tabular}{crrrrrrr}
\hline Classes & LL & AIC & BIC & CAIC & SABIC & BLRT & $p$ \\
\hline 1 & -3519.63 & -7043.26 & -7053.08 & -7055.08 & -7046.73 & --- & --- \\
2 & -3399.82 & $\mathbf{- 6 8 0 9 . 6 4}$ & $\mathbf{- 6 8 3 4 . 1 8}$ & $\mathbf{- 6 8 3 9 . 1 8}$ & $\mathbf{- 6 8 1 8 . 3 0}$ & 239.63 & .001 \\
3 & -3400.23 & -6816.46 & -6855.72 & -6863.72 & -6830.31 & -0.82 & $\mathbf{. 9 4 6}$ \\
4 & -3398.41 & -6872.80 & -6837.86 & -6883.80 & -6818.82 & 3.65 & .135 \\
\hline
\end{tabular}

Note. $\mathrm{LL}=\mathrm{Log}$ Likelihood; note that in mclust higher values on information criteria indicate better fit; boldface $=$ highest value/first non-significant BLRT .

Concerning classification diagnostics, we can use the uncertainty values and modal class assignments stored in the mclust output object to calculate average posterior class probabilities which are $A v e P P_{1}=.95$ for Class 1 and $A v e P P_{2}=.98$ for Class 2 (see SOM). Entropy values can be obtained by get_fit() and show that the 2-class model has high classification accuracy $(E=.89)$. Thus having identified a best fitting model, we can inspect its estimated parameters in the output pasted below.

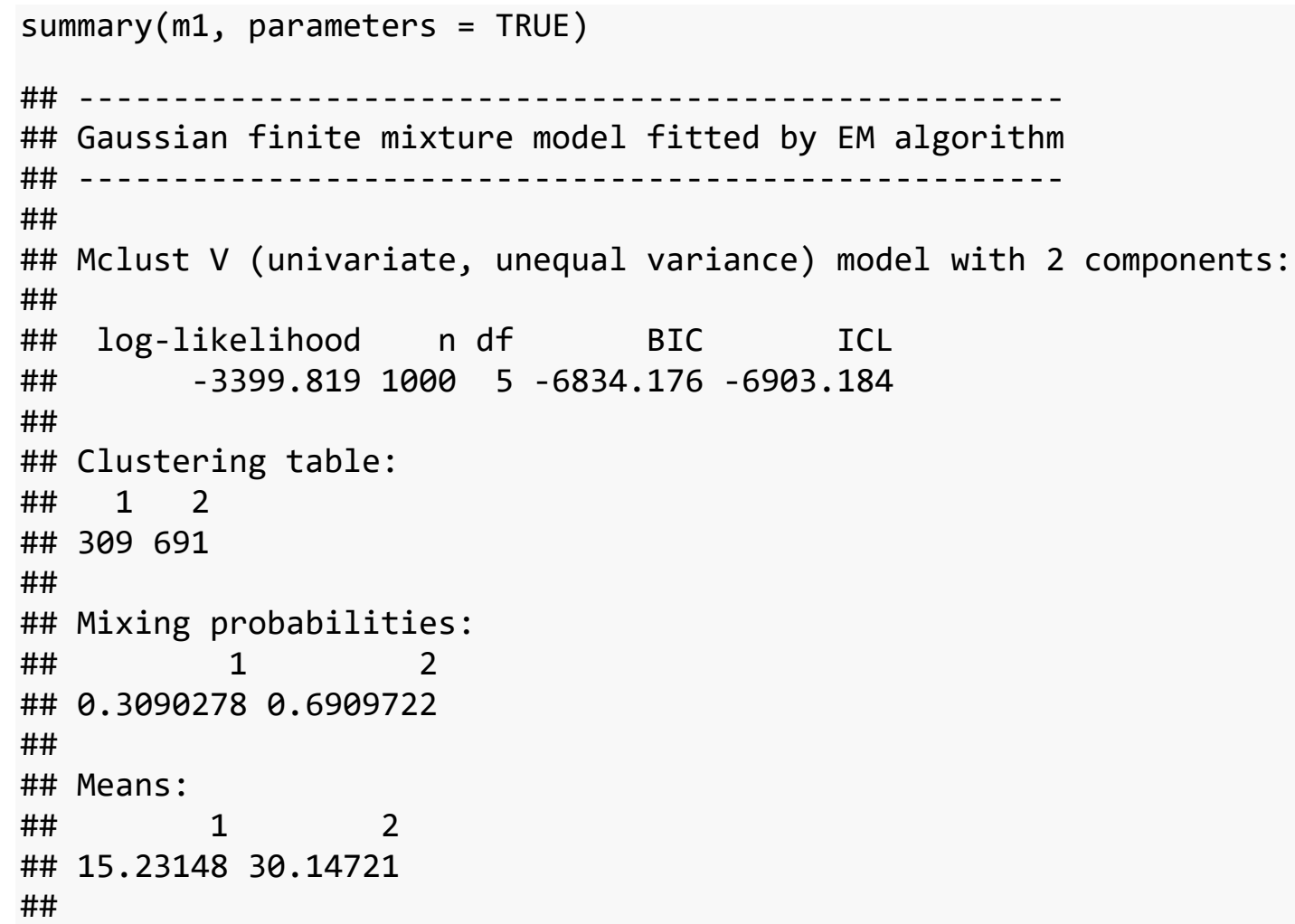


\#\# Variances:

\#\# 112

\#\# $\quad 9.63350123 .584189$

The first part of the output reiterates information about model fit. The clustering table tells us that $n=309$ persons had their modal class membership in Class 1 , and $n=691$ in Class 2 . The estimated class sizes in the population (mixing probabilities) are $30.9 \%$ for Class 1 and $69.1 \%$ for Class 2 . The most important part of the output are the sections on class-specific means and variances. Class 1 has an average achievement of $M=15.2(S D=3.1)$, and Class 2 $M=30.1(S D=4.9)$. Given these values, the two classes can be interpreted as consisting of low-achievers and high-achievers, respectively.

Apparently, our LPA recovered the class-specific population parameters very well (cf. Fig 1b). Of course, LPA cannot deliver evidence that there are two latent classes because some students applied different learning strategies than others. To investigate this, we might conduct a new study collecting additional data on students' learning strategies and checking whether strategies predict latent class membership. We could also collect additional data on some external criterion (say later study success) and use the latent class variable as a predictor.

Finally, with our simulated data, it is possible to compare class assignment based on the LPA results with individuals' true class membership. As table 3 shows, there is a correct classification rate of $97.5 \%$. So, the LPA was quite effective in recovering the unobserved groups.

Table 3. True class versus most likely class membership from LPA.

\begin{tabular}{|c|c|c|c|}
\hline \multirow[b]{2}{*}{ True } & \multicolumn{2}{|c|}{ LPA } & \multirow[b]{2}{*}{ Total } \\
\hline & 1 & 2 & \\
\hline $\mathrm{A}$ & 292 & 8 & 300 \\
\hline B & 17 & 683 & 700 \\
\hline Total & 309 & 691 & 1000 \\
\hline
\end{tabular}


This example of a univariate LPA demonstrated general procedures that transfer to the multivariate case. Though univariate LPA may rarely be of substantive interest in applied research, it can be a useful methodological tool. For example, in a recent online study, we used it to identify a cluster of participants with unreasonably short time on task that we discarded as junk participations. LPA provides arguably a more principled approach for this purpose than setting an arbitrary cut off time. Moreover, univariate LPA may be an alternative to common grouping procedures, such as the median split (Rost, 2006). For this purpose, equality restrictions may be imposed on the class sizes to yield equally sized groups.

\subsection{Example 2: Multivariate LPA of work recovery}

In most applied cases researchers will wish to use LPA on multiple indicators. The illustrative example presented in this section is based upon Study 1 in Gillet et al. (2020) who investigated profiles of work recovery (and related predictors) in a sample of $N=415$ employees from various occupations. Factor scores on three psychological mechanisms of work recovery served as indicators: (i) psychological detachment as the ability to mentally step back from work after hours; (ii) rumination as a tendency to repetitively or continuously be preoccupied with distressful aspects after work; and (iii) overcommitment as a generic tendency to excessively strive for achievement and recognition at work. Whereas psychological detachment is a functional mechanism for the work recovery process, rumination and overcommitment are dysfunctional ones. Population values for the data simulation $(N=500)$ were chosen in line with results from Gillet et al. (2020). Hence, within sampling error, our analysis will lead to roughly identical conclusions.

The code below instructs mclust to specify and fit a series of models with $k=1$ to 8 latent classes. As mentioned above, if we specify no particular model restrictions the software estimates a set of 14 models that impose specific model constraints based on different geometric features of $\boldsymbol{\Sigma}_{\boldsymbol{k}}$ (Scrucca et al., 2016). The substantive implications of these features may be hard 
to interpret for applied researchers. Important models, also discussed in other literature on LPA (Masyn, 2013; Pastor et al., 2007; Rosenberg et al., 2018), are in order of decreasing parsimony:

- EEI: variances equal across classes, covariances fixed at zero

- VVI: class-varying variances, covariances fixed at zero

- EEE: variances and covariances equal across classes

- VVV: class-varying variances and covariances

Of these, EEI and VVI are local independence models, EEE and VVV relax this assumption.

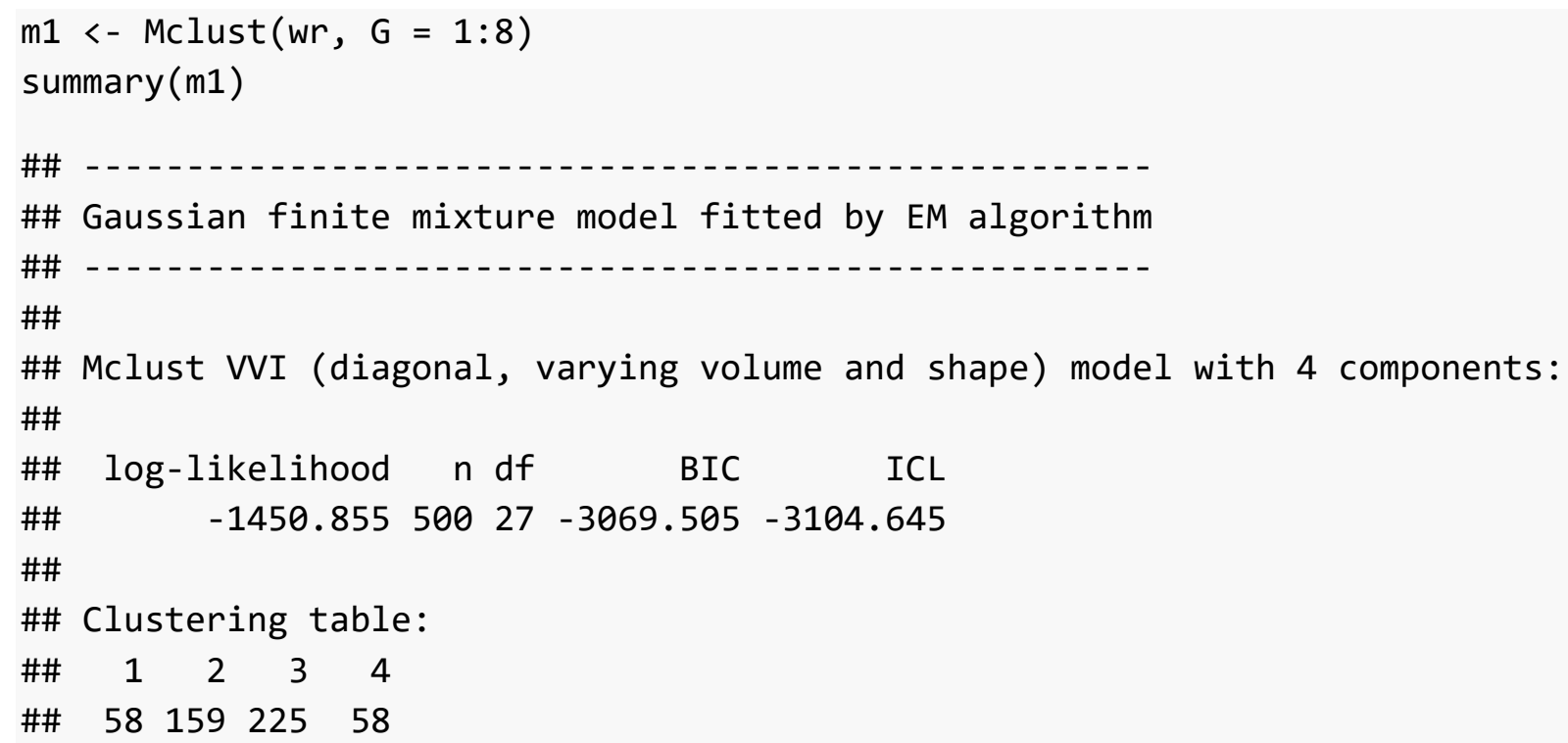

The summary output suggests that the VVI model with four latent classes fits the data best. To inspect fit more closely, we can call for an elbow-plot comparing all mclust models (see SOM). Of course, as an alternative to this data-driven strategy, we could have specified a priori parameter restrictions on $\boldsymbol{\Sigma}_{\boldsymbol{k}}$ based on substantive considerations. For example, we could have specified local independence and equal cross-class variances by setting the argument modelName = "EEI". To test the tenability of these restrictions, next, we could compare the fit of the EEI model to a less restrictive one such as VVI.

As demonstrated in the univariate example, it may be desirable to base the class enumeration decision on a broader set of indices. Again, this is achieved conveniently using tidyLPA. The function compare_solutions () from this package delivers a combined 
recommendation based on a number of fit indices (see SOM). Moreover, it may be helpful to plot selected indices. As visible from Figure 2, the four-class solution fits best according to BIC, CAIC. AIC and SABIC seem less conclusive but parsimony would speak for the fourclass solution, too, unless a model with more classes would have advantages in terms of substantive interpretability. The BLRT pasted in the output below adds further support to the four-class solution by getting non-significant for the comparison of 5 versus 4 classes.

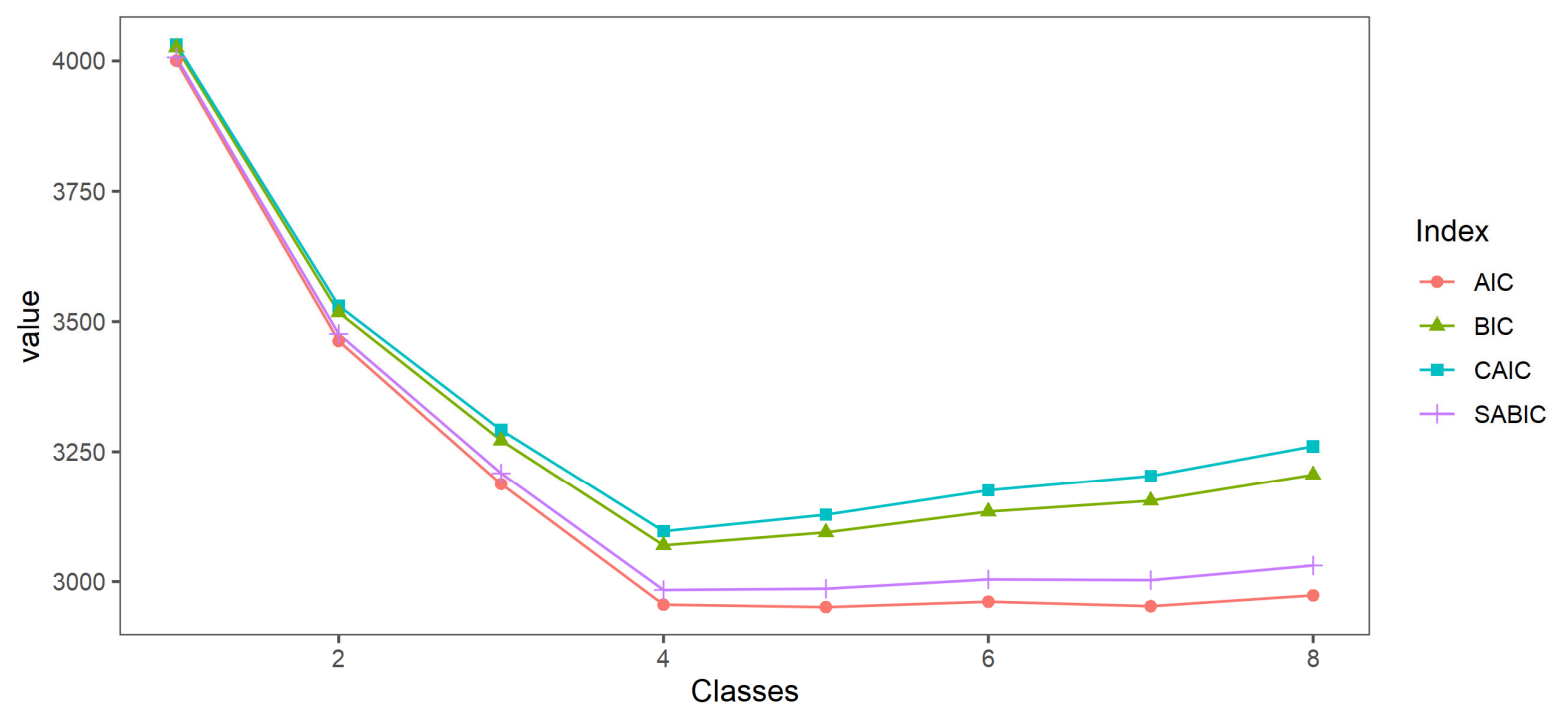

Fig. 2. Elbow-plot of selected fit indices for the multivariate LPA.

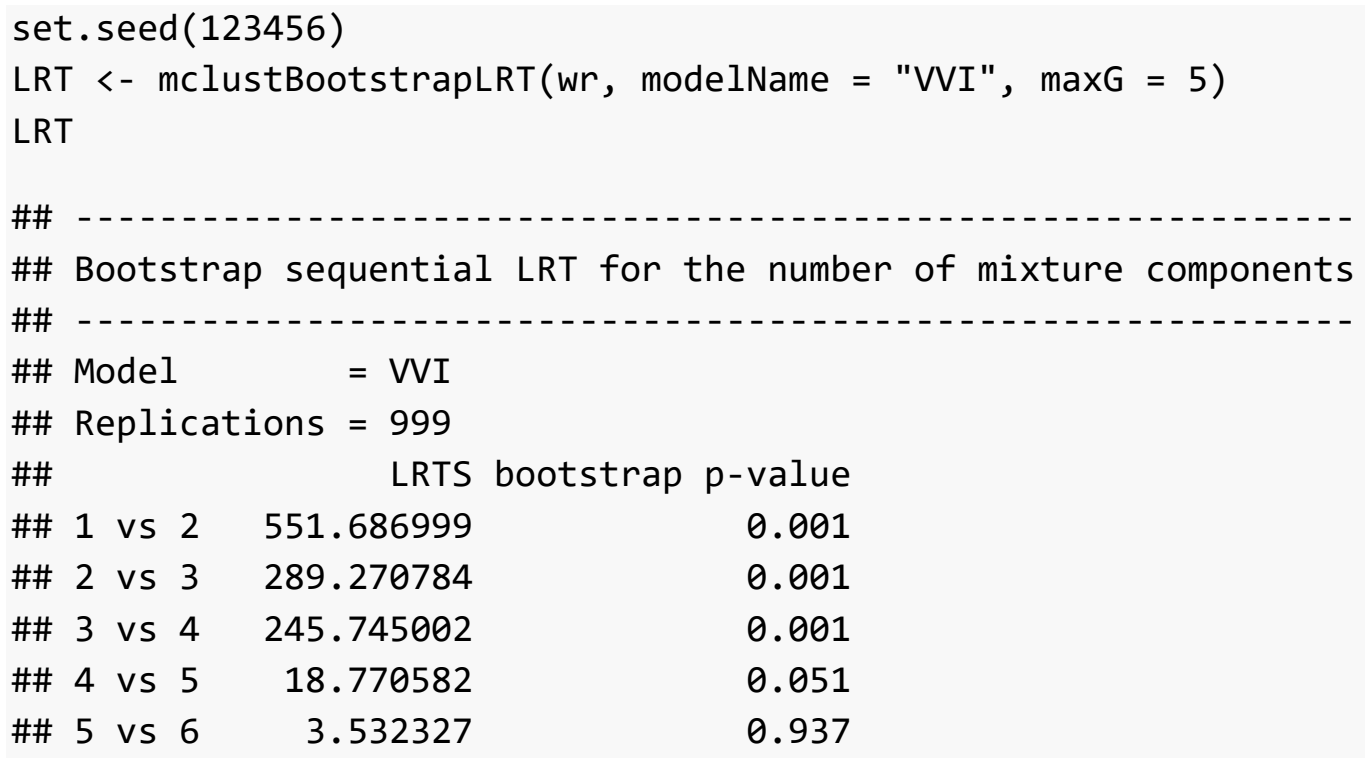


Next, we inspect classification diagnostics. The listing below delivers AvePP values all of which are satisfactory. Entropy is high, too $(E=.93$; see SOM). mclust offers additional diagnostic plots based on dimensionality reduction that allow inspecting the boundaries between the classes to see whether they are well separated (SOM).

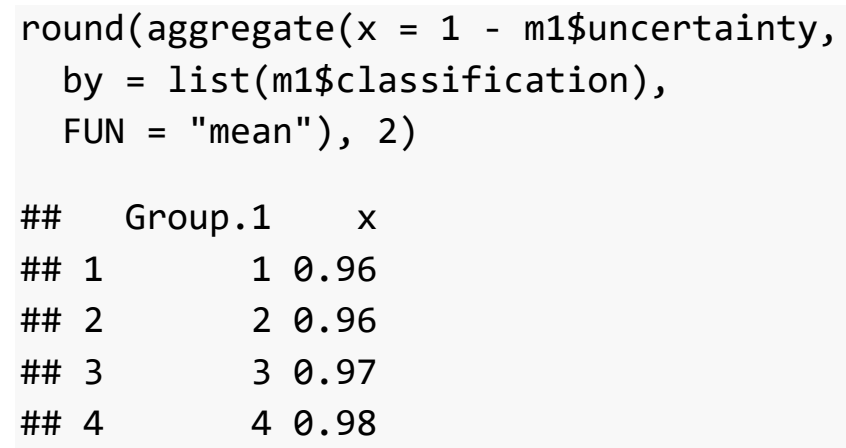

Having decided on the number of classes, we can refit the target model and inspect the model results. Below is a shortened output.

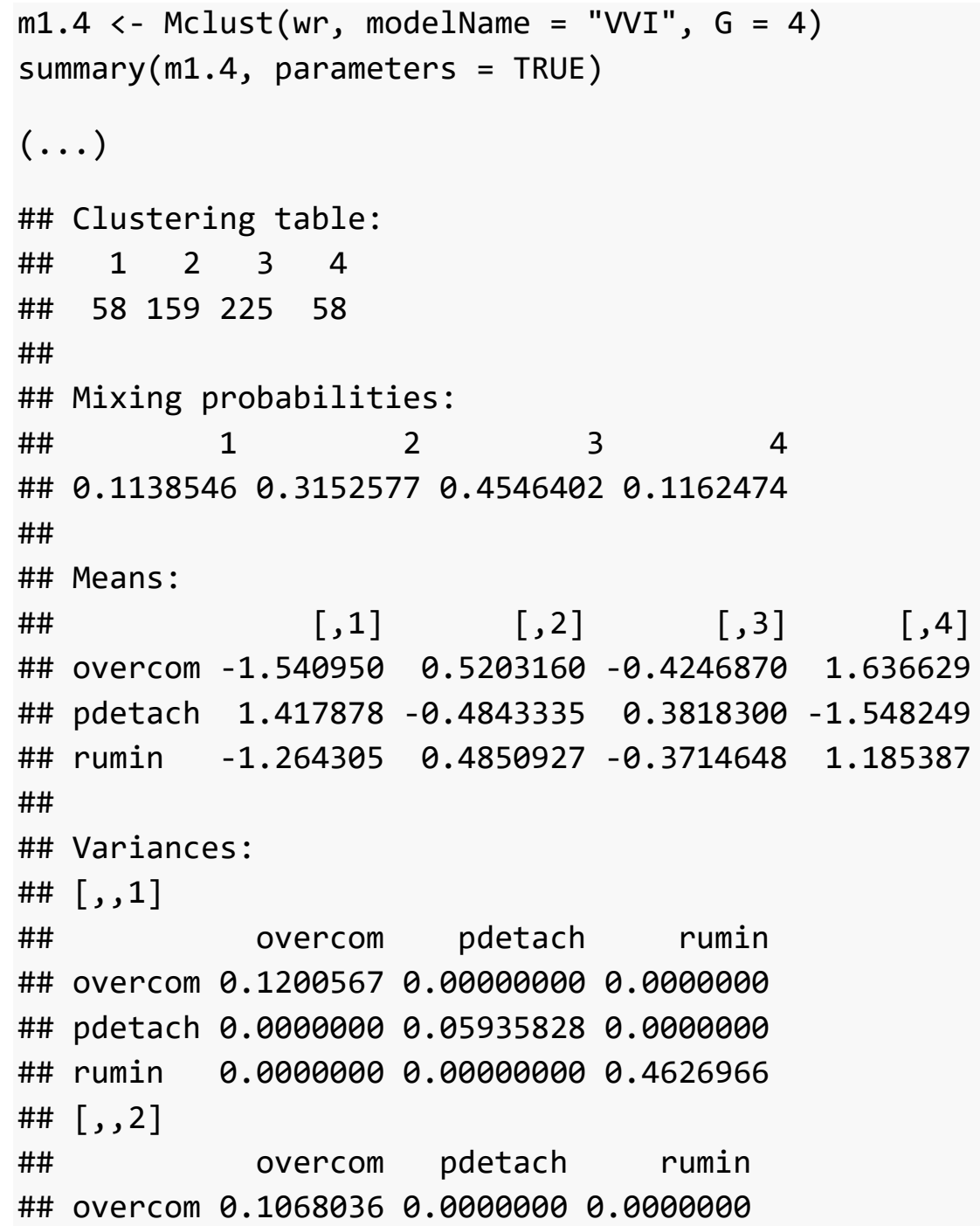


\#\# pdetach $0.0000000 \quad 0.14904780 .0000000$

\#\# rumin $0.0000000 \quad 0.0000000 \quad 0.1430751$

$(\ldots)$

The mixing probabilities indicate that class sizes vary between 11 and $45 \%$ of the sample. The class-specific mean profiles can be found under "Means". Plotting them facilitates their interpretation (Fig. 3). As concluded in Gillet et al. (2020), the classes can be interpreted to demonstrate high (Class 1, $11 \%$ ), moderately high (Class 3, $45 \%$ ), moderately low (Class 2, $32 \%$ ), and low (Class 4, $11 \%$ ) ability to achieve work recovery. Note that this implies that the identified latent classes differ mostly in terms of degree rather than by type. Indeed, the profiles would be perfectly ordered had we recoded psychological detachment so that higher values on all three indicators meant worse ability to achieve work recovery. As discussed above, such an ordered classification is meaningful by pointing at clusters of persons who show similar levels of the investigated trait(s). Nevertheless, these quantitative differences could be modeled more parsimoniously using a continuous latent variable model (Rost, 2006; Wang \& Wang, 2020). So, some might question whether LPA is the best modeling choice in the present case.

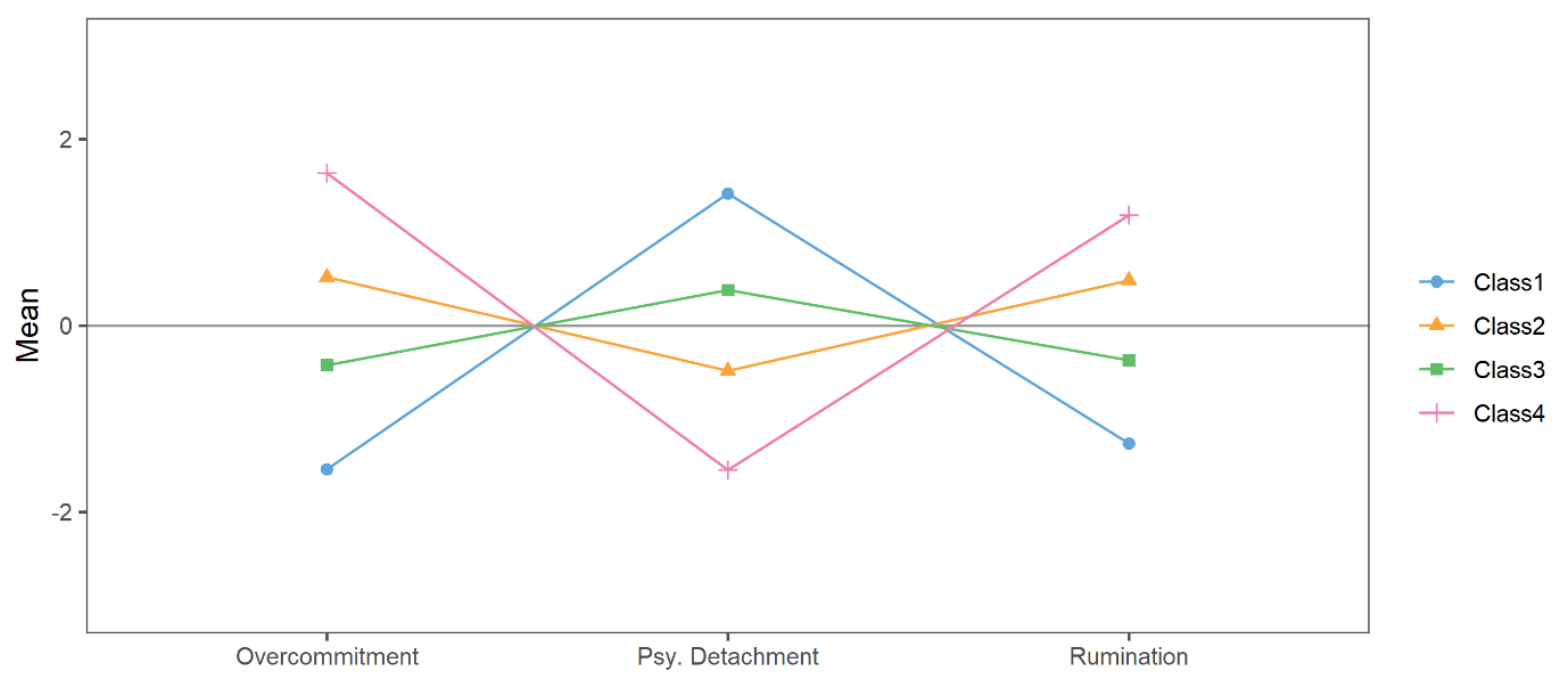

Fig. 3. Mean profiles of four latent classes of work recovery (simulated data based on Gillet et al., 2020). 
The last part of the output shows the class specific variance-covariance matrices. As implied by the VVI model, the class-specific covariances are fixed at zero (i.e. local independence) and the indicator variances are allowed to vary. For example, the variance of rumination is higher in Class 1 than in Class 2. Such information may be important for understanding the nature of the latent classes.

Finally, a comparison with individuals' true population class membership shows that about $97 \%$ of our sample have been classified correctly by the LPA (SOM).

\subsection{Example 3: LCA of teachers' participation in professional development}

This section gives an applied example of LCA for categorical variables using the poLCA package. It draws upon a study by Richter et al. (2013) who investigated individual differences in language teachers' $(N=2076)$ participation in professional development (PD) programs. In their LCA, the authors analyzed eight dichotomized items indicating whether teachers during the last five years had participated (yes/no) in PD activities on each of the following topics: (i) content knowledge, and (ii) pedagogical content knowledge, each with a focus on reading comprehension, (iii) teaching and learning methods, (iv) teaching heterogeneous students, (v) classroom management and discipline, (vi) individualized learning support, (vii) counseling students and parents, and (viii) knowledge about kids and youth. As in Example 2, we will use simulated data $(N=2076)$ with population parameters based on the outcomes of the original study.

For model specification, poLCA requires users to explicitly provide a model formula. The first part of the code below specifies a LCA-model with no covariates and stores it in an R object. Next, we use it to estimate a series of models with $k=1$ to 7 latent classes. Unlike in mclust, each model needs to be estimated in a separate function call. The argument nrep specifies the number of times to estimate each model using different sets of random starting values for the estimation algorithm. This is important to avoid solutions that converge at local instead of global maxima of the likelihood function. Local maxima are a plague in mixture 
modeling (e.g. Masyn, 2013). We did not need to take care of this problem in the previous LPA examples because mclust employs a different approach to avoid local maxima (Scrucca \& Raferty, 2015). In the present LCA, we estimate each model with at least ten sets of random starting values. Larger numbers are typically required for complex models, especially in serious applications. Here, we increase the number of random starts for the models with 6 and 7 latent classes and additionally increase the maximum number of iterations (default $=1000)$ to attain convergence of the EM algorithm.

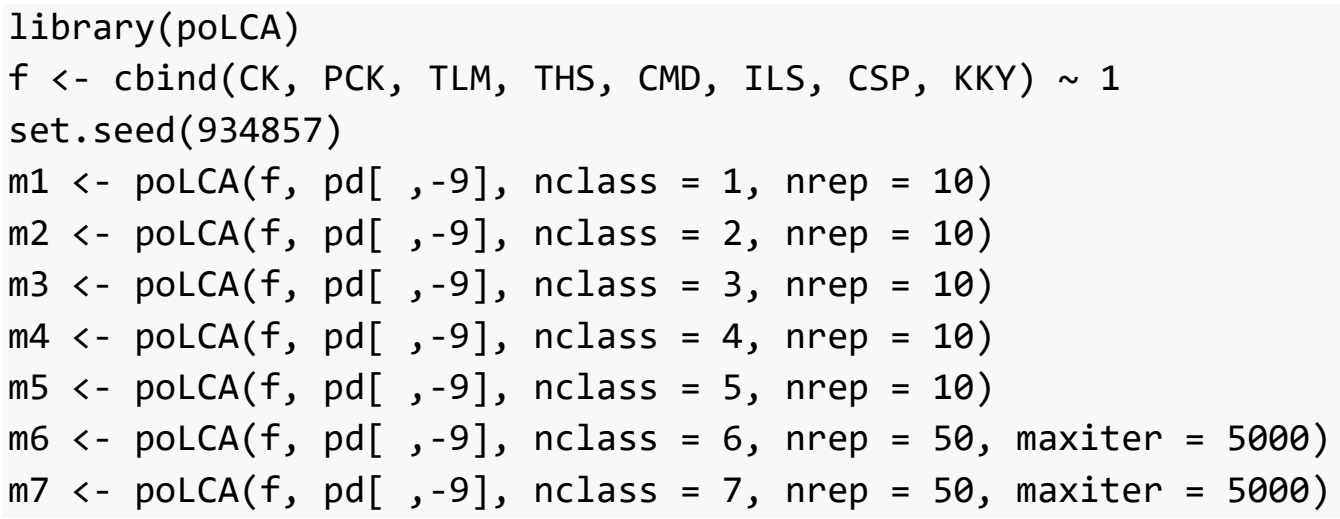

To assess model fit, poLCA delivers the information criteria AIC and BIC. As in the previous examples, further fit indices can be calculated manually based on the model results (SOM). The elbow plot in Figure 4 shows that BIC, CAIC, and SABIC point at a best fitting solution with five latent classes whereas AIC continues to improve as more classes are extracted. Unfortunately, the BLRT is not available in poLCA. So, based on the present information, we may preliminarily decide for the 5-class model and inspect classification diagnostics. 


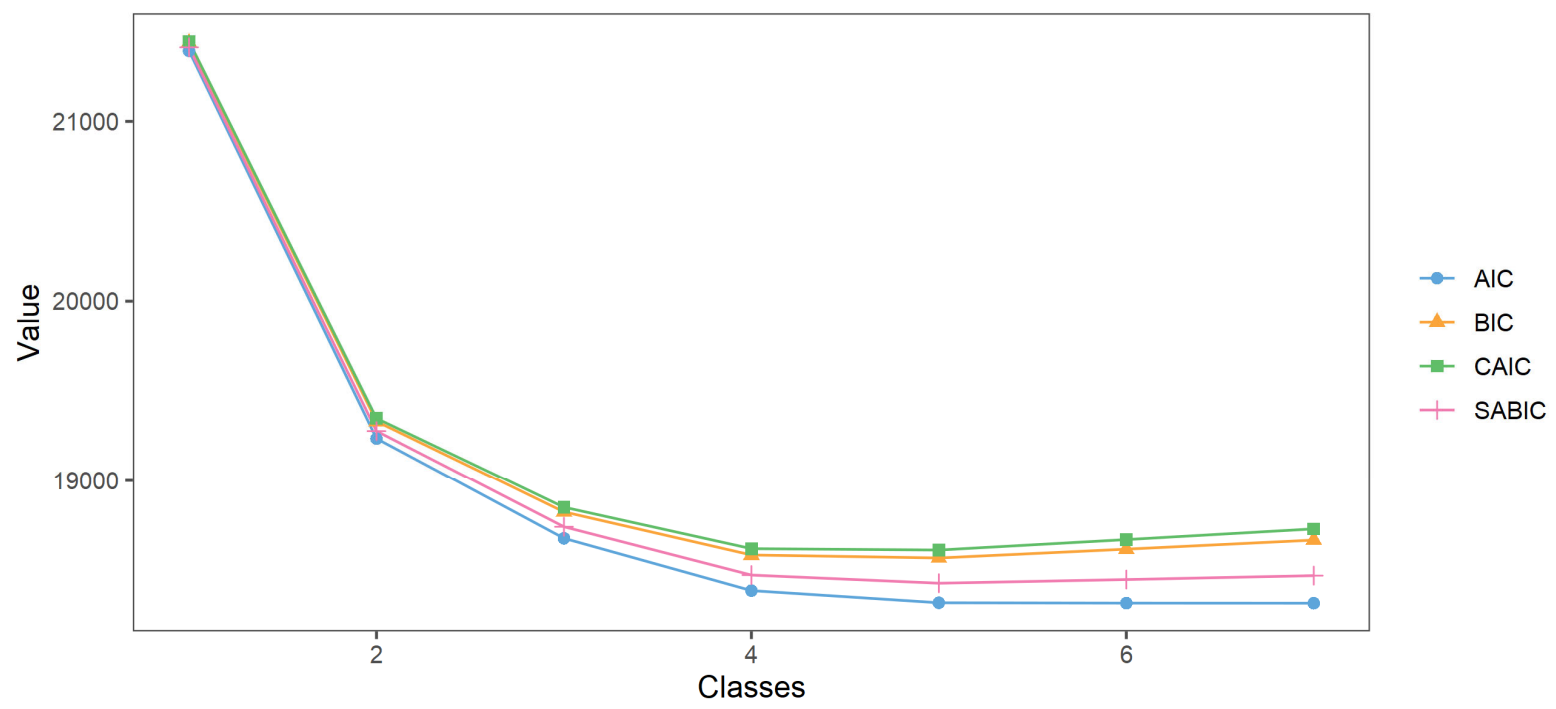

Fig. 4. Elbow-plot of selected fit indices for the LCA.

The listing below, first calculates $A v e P P$ all of which are $>.70$. Regarding entropy, note that poLCA does not estimate relative entropy as is typically used in other software. The function provided below provides this index (cf. Collins \& Lanza, 2010, p. 75). As we can obtain from the output, entropy for the 5-class model is somewhat below the recommended value of .80 but acceptable (i.e., $>.60$ ). From the series of values, we can see that initially entropy decreases slightly as more classes are extracted. As Collins and Lanza (2010) note, this is frequently the case and an artefact of chance. Recall from the discussion above that entropy is not a good indicator for class enumeration.

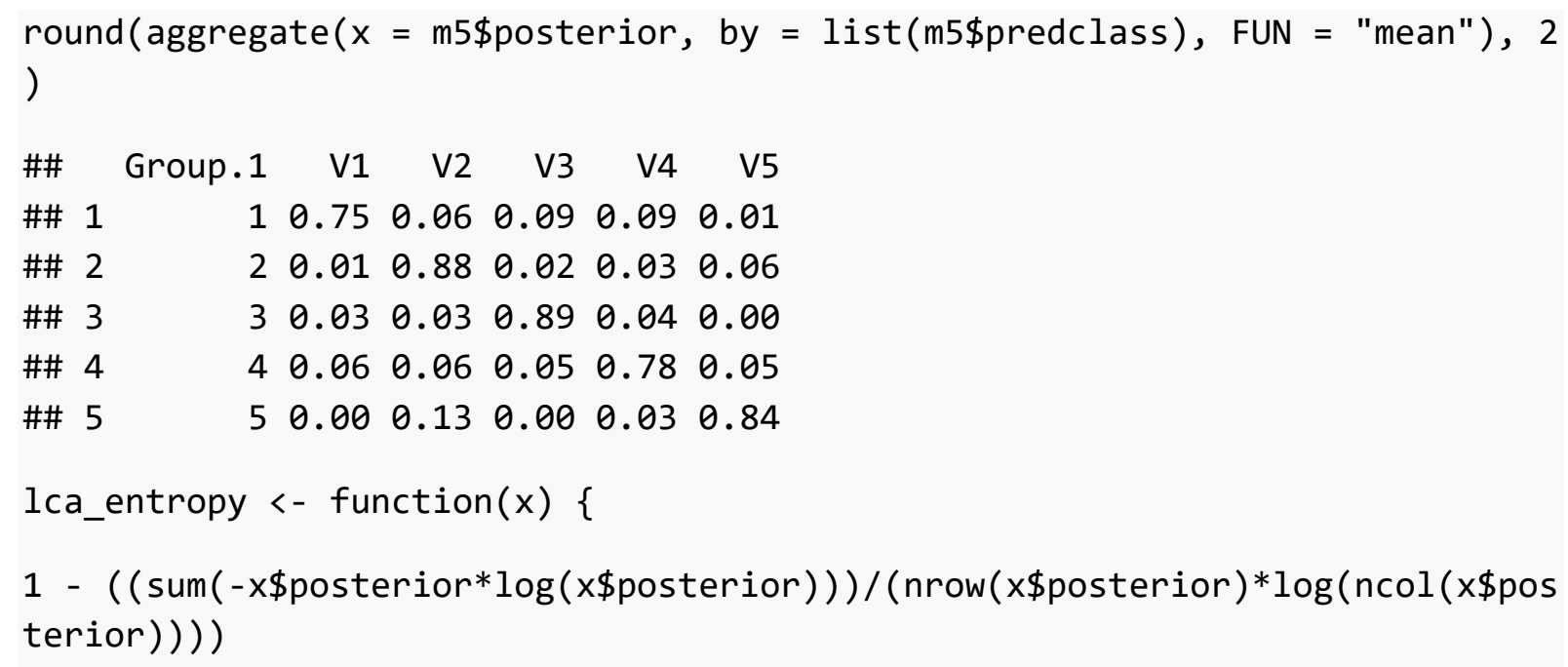






Having decided for the 5-class model, we can inspect the model parameters. Below is a shortened output.

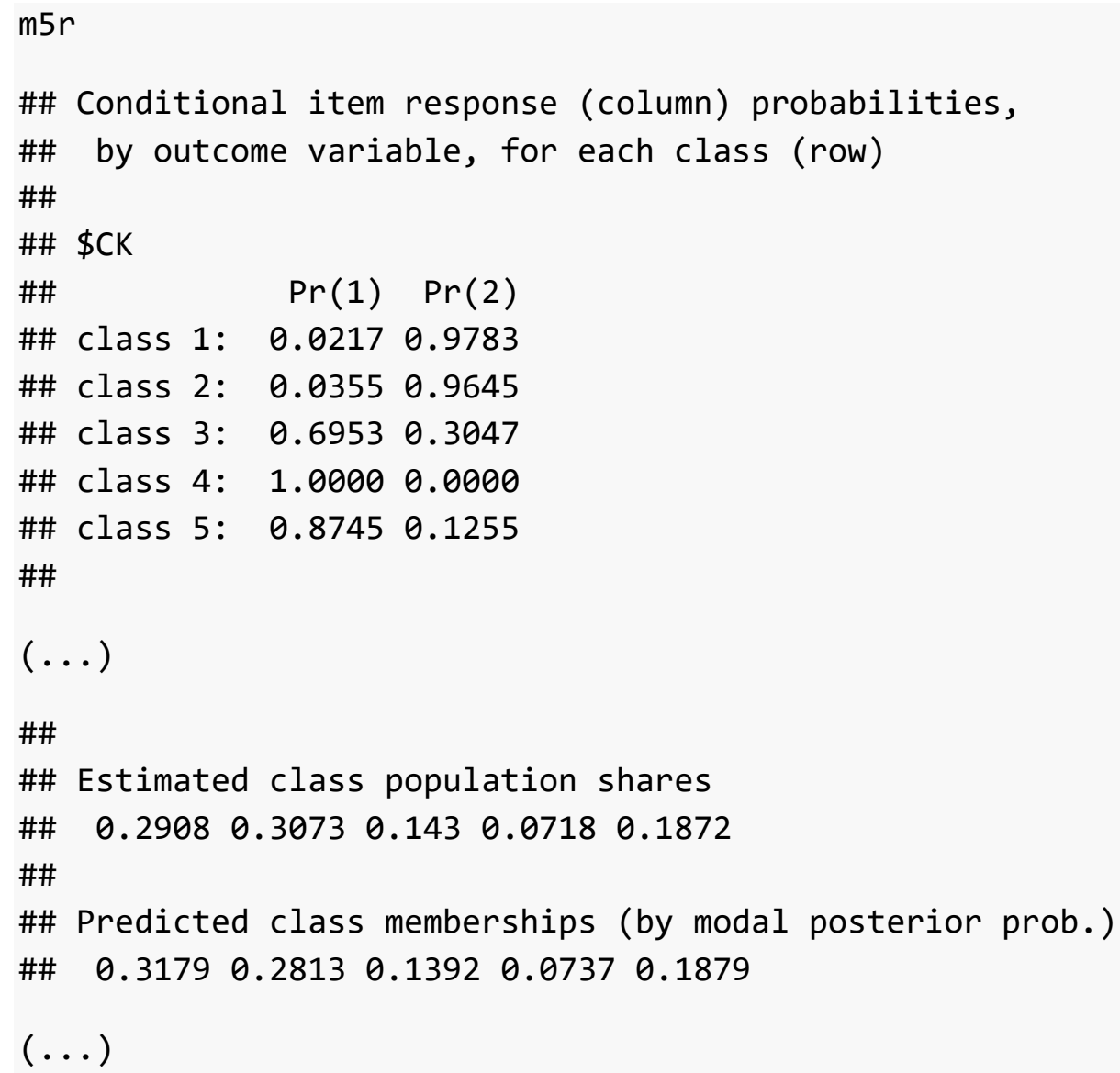

In the first part of the output, poLCA gives us the class-specific response probabilities per indicator and category. For example, teachers in Class 1 have a $98 \%$ probability of having attended PD focusing on content knowledge, whereas teachers in Class 5 have only a $13 \%$ probability. Next, the output contains the latent class sizes in two forms: as estimated class population shares and based on modal class assignment. Both types of values are quite close as they should be. For class interpretation, poLCA provides a 3D-barplot of the conditional item response probabilities (SOM). This plot is hard to read, however, especially with growing numbers of classes, indicators, and answer categories. Since we analyzed dichotomous 
variables, a profile plot of the conditional item probabilities is better suited for interpretation (Fig. 5).



Fig. 5. Conditional item probabilities of five latent classes of participation in professional development activities (simulated data and adapted figure based on Richter et al., 2013); $\mathrm{CK}=$ content knowledge, PCK = pedagogical content knowledge, TLM $=$ teaching and learning methods, THS = teaching heterogeneous students, CMD = classroom management and discipline, ILS = individualized learning support, $\mathrm{CSP}=$ counseling students and parents, KKY $=$ knowledge about kids and youth .

Following Richter et al. (2013) the classes can be interpreted as high on all activities (Class 1,29.1\%), high on activities focusing (pedagogical) content knowledge and teaching methods (Class 2, $30.7 \%$ ), high on activities focusing general pedagogical topics (Class 3, $14.3 \%$ ), high on activities focusing teaching methods (Class 4, $7.2 \%$ ), and low on all activities (Class $5,18.7 \%)$. Note that, due to sampling error, the class sizes estimated from our simulated data deviate somewhat from Richter et al. (2013). 
Two more points are noteworthy. First, the order of classes in any mixture model is arbitrary and, thus, may change over a series of models, software used etc. That is, Class 1 from a given solution, may be Class $k$ in another. The order of classes can be adjusted by providing respective starting values to the estimation algorithm (SOM). In the above output, classes are reordered to match results reported in Richter et al. (2013). Second, a closer inspection the output reveals that Class 4 has boundary conditional probabilities on the indicator CK ( 1 and 0 for no and yes, respectively). Finding many such boundary estimates may be a warning sign that the model solution is invalid or that too many classes have been extracted (Geiser, 2013). Even a single one should be a cause for caution. In general, boundary values may be legitimate estimates but they can also be an indication of model misspecification. In the present example, the respective population value for the simulation was $p=.98$ which already is close to the upper bound of probability. So, within sampling error, the result is a reasonable estimate.

Finally, a check of how well LCA recovered individuals' true class membership shows only an $85 \%$ correct classification rate with misclassification being particularly apparent for Class 4 (SOM). This reduced accuracy may be due to the classes not being well separated from the outset, as hinted by the suboptimal entropy value. Hence, this example may serve as a caveat that even in solutions that seem reasonable in terms of fit and interpretation a non-trivial degree of misclassification can be prevalent.

\section{Conclusion}

This chapter provided an applied introduction to LPA/LCA and their implementation in statistical software using simulated examples of real research. As demonstrated, LPA/LCA are effective analytic techniques for identifying unobserved subgroups that can help researchers to investigate individual differences in learning, among other interests. Modern software as well as methodological advances have greatly facilitated the use of these analytic techniques for applied researchers. 


\subsection{Some words of caution}

Despite the great potential of mixture models some cautionary words are in order. First, as elaborated in this chapter, some aspects of mixture modeling are inevitably exploratory. This should humble researchers in drawing overly strong conclusions from them. In the same vein, readers will have recognized that mixture modeling is a complex process that requires a multitude of decisions and, thus, provides great researcher degrees of freedom. The analytic results can be quite sensitive to these choices and decisions. Therefore, it is crucial to work out a solid and principled plan ahead of the analyses, including a specification of the criteria for the various decisions to make. Otherwise, there is a substantial danger of getting lost in the analytic garden of forking paths.

Second, several fallacies in the interpretation of mixture models are to avoid. As Loken and Molenar (2007) note, “(...) successfully applying a mixture model does not immediately confirm the appropriateness of the model” (p. 278). That is, finding a relatively best fitting model within a series of candidate models neither implies that the model is statistically good in an absolute sense nor that it is a substantively correct representation of the phenomenon in the population. Moreover, results from a mixture model cannot provide sufficient evidence to confirm that the construct of interest indeed is categorical in nature (Loken \& Molenar, 2007; Masyn, 2013). Above I have emphasized that, conceptually, person-centered and variablecentered analyses look at two sides of the same coin. The statistical counterpart of this is that though factor and mixture models are not completely equivalent - "(...) a $k$-group mixture (or latent profile) model can be redescribed as factor model with $k$-1 factors that is identical up to first- and second-order moments" (Loken \& Molenar, 2007, p. 293). Finally, as with other latent variable models, the reification-fallacy should be avoided. Reification occurs when abstract concepts represented by latent variables are taken too literally and are interpreted as if they had a real, physical existence (Agresti, 2019; Bauer, 2007). Just because one successfully identified latent classes and attached labels to them does not mean that these classes exist in the real world. 
In many cases, latent classes should be seen as statistical summaries that identify persons with similar response patterns and, thus, can be helpful interpretative tools. However, such analytic summaries are insufficient evidence to make the case that a specific typology has a concrete reality or that an individual is of a certain naturally occurring type (Bauer, 2007; Masyn, 2013).

\subsection{A note on software and further reading}

There is a wealth of alternatives to the software introduced in this chapter. Many R packages relevant for mixture modeling are listed on the Cran Task Views Psychometric Models and Methods and Clusters (https://cran.r-project.org/web/views). As for proprietary software, Mplus and Latent Gold are easy to use, can estimate a broad array of mixture models, and implement modern approaches of handling covariates and distal outcomes. An applied introduction to mixture modeling using Mplus is available, for example, in Wang and Wang (2020). For Latent Gold, instructive user guides are freely available on the software's website. These and other software options are reviewed in Haughton et al. (2009), Hickendorf et al. (2018), and Uebersax (2012).

For delving more deeply into mixture models, the following selected sources may be of interest: Highly readable introductions can be found in Collins and Lanza (2010), Masyn (2013), and Vermunt and Magidson (2002). Systematic, though more technically challenging, overviews are available in Hancock and Samuelsen (2008), Hancock et al. (2019), McLachlan and Peel (2000), Muthén (2008), and Sterba (2013). The edited volumes by Hagenaars and McCutcheon (2002), and Rost and Langeheine (1997) contain high quality illustrative applications (mostly of LCA) from a broad range of disciplinary and substantive perspectives.

\section{References}

Agresti, A. (2019). An introduction to categorical data analysis. Wiley.

Asparouhov, T., \& Muthén, B. (2014). Auxiliary variables in mixture modeling: Three-step approaches using Mplus. Structural Equation Modeling: A Multidisciplinary Journal, 21(3), 329-341. https://doi.org/10.1080/10705511.2014.915181 
Asparouhov, T., \& Muthén, B. (2015). Residual associations in latent class and latent transition analysis. Structural Equation Modeling: A Multidisciplinary Journal, 22(2), 169-177. https://doi.org/10.1080/10705511.2014.935844

Asparouhov, T., \& Muthén B. (2021). Auxiliary variables in mixture modeling: Using the BCH method in Mplus to estimate a distal outcome model and an arbitrary second model (Mplus Web Notes 21, Version 11). https://www.statmodel.com/MixtureModeling.shtml

Bakk, Z., \& Kuha, J. (2021). Relating latent class membership to external variables: An overview. British Journal of Mathematical and Statistical Psychology, 74(2), 340-362. https://doi.org/https://doi.org/10.1111/bmsp.12227

Bauer, D. J. (2007). Observations on the use of growth mixture models in psychological research. Multivariate Behavioral Research, 42(4), 757-786. https://doi.org/10.1080/00273170701710338

Bauer, J., Gartmeier, M., Wiesbeck, A. B., Moeller, G. E., Karsten, G., Fischer, M. R., \& Prenzel, M. (2018). Differential learning gains in professional conversation training: A latent profile analysis of competence acquisition in teacher-parent and physician-patient communication. Learning and Individual Differences, 61(1), 1-10. https://doi.org/https://doi.org/10.1016/j.lindif.2017.11.002

Bauer, J., \& Mulder, H. R. (2013). Engagement in learning after errors at work: enabling conditions and types of engagement. Journal of Education and Work, 26(1), 99-119. https://doi.org/10.1080/13639080.2011.573776

Bauer, J. \& Prenzel, M. (2021). For what educational goals do preservice teachers feel responsible? On teachers' ethos as professional values. In F. Oser, K. Heinrichs, J. Bauer, \& T. Lovat (Eds.), The international handbook of teacher ethos: Strengthening teachers, supporting learners (pp. xxx). Springer. DOI:XXX 
Bergman, L. R., \& Magnusson, D. (1997). A person-oriented approach in research on developmental psychopathology. Development and Psychopathology, 9(2), 291-319. https://doi.org/10.1017/S095457949700206X

Collins, L. M., \& Lanza, S. T. (2010). Latent class and latent transition analysis. Wiley.

Finch, W. H., \& Bronk, K. C. (2011). Conducting confirmatory latent class analysis using Mplus. Structural Equation Modeling: A Multidisciplinary Journal, 18(1), 132-151. https://doi.org/10.1080/10705511.2011.532732

Geiser, C. (2013). Data analysis with Mplus. Guilford.

Gillet, N., Morin, A. J. S., Mokounkolo, R., Réveillère, C., \& Fouquereau, E. (2020). A personcentered perspective on the factors associated with the work recovery process. Anxiety, Stress, \& Coping, 1-26. https://doi.org/10.1080/10615806.2020.1866174

Hagenaars, J. A., \& McCutcheon, A. L. (Eds.). (2002). Applied latent class analysis. Cambridge University Press.

Hancock, G. R., Harring, J. R., \& Macready, G. B. (Eds.). (2019). Advances in latent class analysis. IAP.

Hancock, G. R., \& Samuelsen, K. M. (Eds.). (2008). Advances in latent variable mixture models. IAP.

Haughton, D., Legrand, P., \& Woolford, S. (2009). Review of three latent class cluster analysis packages: Latent Gold, poLCA, and MCLUST. The American Statistician, 63(1), 8191. https://doi.org/10.1198/tast.2009.0016

Hickendorff, M., Edelsbrunner, P. A., McMullen, J., Schneider, M., \& Trezise, K. (2018). Informative tools for characterizing individual differences in learning: Latent class, latent profile, and latent transition analysis. Learning and Individual Differences, 66, 415. https://doi.org/https://doi.org/10.1016/j.lindif.2017.11.001

Hong, W., Bernacki, M. L., \& Perera, H. N. (2020). A latent profile analysis of undergraduates' achievement motivations and metacognitive behaviors, and their relations to 
achievement in science. Journal of Educational Psychology, 112(7), 1409-1430. https://doi.org/10.1037/edu0000445

Jung, T., \& Wickrama, K. A. S. (2008). An introduction to latent class growth analysis and growth mixture modeling. Social and Personality Psychology Compass, 2(1), 302-317. https://doi.org/https://doi.org/10.1111/j.1751-9004.2007.00054.x

Kunst, E. M., van Woerkom, M., \& Poell, R. F. (2018). Teachers' goal orientation profiles and participation in professional development activities. Vocations and Learning, 11(1), 91111. https://doi.org/10.1007/s12186-017-9182-y

Linzer, D. A., \& Lewis, J. B. (2011). poLCA: An R package for polytomous variable latent class analysis. Journal of Statistical Software, 42(10), 1-29. https://doi.org/10.18637/jss.v042.i10

Little, T. D., Slegers, D. W., \& Card, N. A. (2006). A non-arbitrary method of identifying and scaling latent variables in SEM and MACS models. Structural Equation Modeling: A Multidisciplinary Journal, 13(1), 59-72. https://doi.org/10.1207/s15328007sem1301_3

Loken, E., \& Molenaar, P. (2008). Categories or continua? The correspondence between mixture models and factor models. In G. R. Hancock, \& K. M. Samuelsen (Eds.), Advances in latent variable mixture models (pp. 277-297). IAP.

Mair, P. (2018). Modern psychometrics with R. Springer. https://doi.org/10.1007/978-3-319$93177-7$

Masyn, K. (2013). Latent class analysis and finite mixture modeling. In T. D. Little (Ed.), The Oxford handbook of quantitative methods in psychology (Vol. 2, pp. 551-611). Oxford University Press.

McLachlan G. J. \& Peel D. (2000). Finite mixture models. Wiley.

Muthén, B. (2008). Latent variable hybrids: Overview of old and new models. In Hancock, G. R., \& Samuelsen, K. M. (Eds.), Advances in latent variable mixture models (pp. 1-24). IAP. 
Muthén, L. K., \& Muthén, B. O. (1998-2017). Mplus user's guide ( $8^{\text {th }}$ ed.). Muthén \& Muthén.

Nylund, K. L., Asparouhov, T., \& Muthén, B. O. (2007). Deciding on the number of classes in latent class analysis and growth mixture modeling: a monte carlo simulation study. $\begin{array}{lll}\text { Structural } \quad \text { Equation } \quad \text { Modeling, } & \text { 14(4), }\end{array}$ https://doi.org/10.1080/10705510701575396

Nylund-Gibson, K., Grimm, R. P., \& Masyn, K. E. (2019). Prediction from latent classes: A demonstration of different approaches to include distal outcomes in mixture models. Structural Equation Modeling: A Multidisciplinary Journal, 26(6), 967-985. https://doi.org/10.1080/10705511.2019.1590146

Oberski, D. (2016). Mixture models: Latent profile and latent class analysis. In J. Robertson \& M. Kaptein (Eds.), Modern statistical methods for HCI (pp. 275-287). Springer. https://doi.org/10.1007/978-3-319-26633-6_12

Pastor, D. A., Barron, K. E., Miller, B. J., \& Davis, S. L. (2007). A latent profile analysis of college students' achievement goal orientation. Contemporary Educational Psychology, 32(1), 8-47. https://doi.org/https://doi.org/10.1016/j.cedpsych.2006.10.003

Peugh, J., \& Fan, X. (2013). Modeling unobserved heterogeneity using latent profile analysis: A monte carlo simulation. Structural Equation Modeling: A Multidisciplinary Journal, 20(4), 616-639. https://doi.org/10.1080/10705511.2013.824780

R Core Team (2021). R: A language and environment for statistical computing. R Foundation for Statistical Computing, Vienna, Austria. https://www.R-project.org

Richter, D., Engelbert, M., Weirich, S., \& Pant, H. A. (2013). Differentielle Teilnahme an Lehrerfortbildungen und deren Zusammenhang mit professionsbezogenen Merkmalen von Lehrkräften [Differential use of professional development programs and its relationship to professional characteristics of teachers]. Zeitschrift für Pädagogische Psychologie, 27(3), 193-207. https://doi.org/10.1024/1010-0652/a000104 
Rosenberg et al., (2018). tidyLPA: An R package to easily carry out latent profile analysis (LPA) using open-source or commercial software. Journal of Open Source Software, 3(30), 978. https://doi.org/10.21105/joss.00978

Rost, J. (2003). Latent class analysis. In R. Fernandez-Ballesteros (Ed.), Encyclopedia of psychological assessment (Vol. 1, pp. 539-543). Sage.

Rost, J. (2006). Latent-Class-Analyse. In F. Petermann \& M. Eid (Eds.), Handbuch der psychologischen Diagnostik (pp. 275-287). Hogrefe.

Rost, J., \& Langeheine, R. (Eds.). (1997). Applications of latent trait and latent class models in the social sciences. Waxmann.

Scrucca, L., Fop, M., Murphy, T. B., \& Raftery, A. E. (2016, Aug). mclust 5: Clustering, classification and density estimation using Gaussian finite mixture models. The $R$ Journal, 8(1), 289-317. https://doi.org/10.32614/RJ-2016-021

Scrucca, L., \& Raftery, A. E. (2015). Improved initialisation of model-based clustering using Gaussian hierarchical partitions. Advances in Data Analysis and Classification, 9(4), 447460. https://doi.org/10.1007/s11634-015-0220-z

Sterba, S. K. (2013). Understanding linkages among mixture models. Multivariate Behavioral Research, 48(6), 775-815. https://doi.org/10.1080/00273171.2013.827564

Tofighi, D., \& Enders, C. K. (2008). Identifying the correct number of classes in growth mixture models. In G. R. Hancock \& K. M. Samuelsen (Eds.), Advances in latent variable mixture models (pp. 317-341). IAP.

Uebersax, J. (2012). LCA software. http://john-uebersax.com/stat/soft.htm

Vermunt, J. K., \& Magidson, J. (2002). Latent class cluster analysis. In J. A. Hagenaars \& A. L. McCutcheon (Eds.), Applied latent class analysis (pp. 89-106). Cambridge University Press.

Vermunt, J. K., \& Magidson, J. (2016). Technical guide for Latent GOLD 5.1: Basic, Advanced, and Syntax. Statistical Innovations Inc. 
Wang, J., \& Wang, X. (2020). Structural equation modeling: Applications using Mplus (2nd ed.). Wiley. 